\title{
Contribución de la comunicación institucional de la investigación a su impacto y visibilidad. Caso de la Universidad Carlos III de Madrid
}

\author{
Contribution of the institutional communication of \\ research to its impact and visibility: the case of the \\ Universidad Carlos III de Madrid
}

\author{
Francisco-Javier Alonso-Flores; Daniela De-Filippo; Antonio-Eleazar Serrano-López; \\ Carolina Moreno-Castro
}

Cómo citar este artículo:

Alonso-Flores, Francisco-Javier; De-Filippo, Daniela; Serrano-López Antonio-Eleazar; Moreno-Castro, Carolina (2020). "Contribución de la comunicación institucional de la investigación a su impacto y visibilidad. Caso de la Universidad Carlos III de Madrid". Profesional de la información, v. 29, n. 6, e290633.

https://doi.org/10.3145/epi.2020.nov.33

Artículo recibido el 10-01-2020 Aceptación definitiva: 13-08-2020

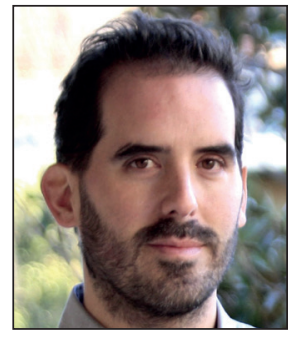

Francisco-Javier Alonso-Flores https://orcid.org/0000-0003-4696-4684

Universidad Carlos III de Madrid, Servicio de Comunicación Institucional Madrid, 12 28903 Getafe (Madrid), España fcojavier.alonso@uc3m.es $\$

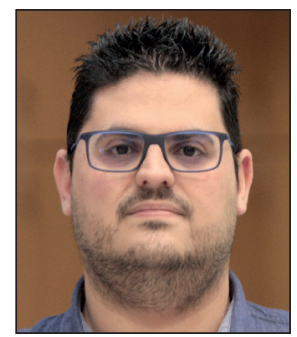

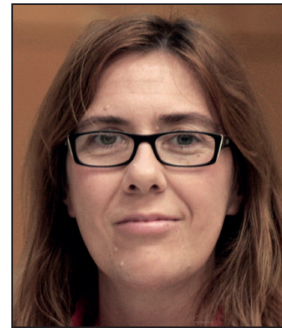

Daniela De-Filippo https://orcid.org/0000-0001-9297-9970

Universidad Carlos III de Madrid, Departamento de Biblioteconomía y Documentación

Madrid, 12

28903 Getafe (Madrid), España dfilippo@bib.uc3m.es

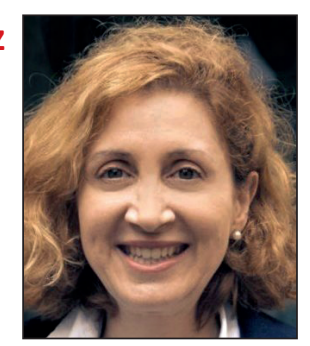

Moreno-Castro https://orcid.org/0000-0001-7453-4257 Universitat de València, Departamento de Teoría de los Lenguajes y Ciencias de la Comunicación

Avda. Blasco Ibañez, 32 46010 Valencia, España carolina.moreno@uv.es

\section{Resumen}

Las oficinas de comunicación y divulgación pública de la ciencia están ganando importancia en las universidades españolas y las actividades que realizan pueden contribuir al incremento de la visibilidad y reputación del personal de investigación. El objetivo de este estudio es analizar las relaciones entre la comunicación institucional pública de los resultados de investigación y el impacto y visibilidad académica de las publicaciones científicas. Para ello, se ha seleccionado como estudio de caso la comunicación y diseminación que realiza la Unidad de Cultura Científica y de Innovación (UCC+i) de la Universidad Carlos III de Madrid. Se ha escogido esta UCC+i porque publica anualmente, desde hace más de una década, informes sobre el impacto que generan en medios de comunicación las notas de prensa que difunden. Se han examina-

Financiación

Este proyecto ha recibido financiación del proyecto "SciSHop-eu" (№ 741657) de la convocatoria Horizonte 2020 de la Unión Europea. El contenido del artículo no refleja la opinión oficial del organismo financiador, sino que queda bajo la exclusiva responsabilidad de los autores. Además, ha contado con la colaboración del Grupo de investigación en Cultura Científica-ScienceFlows (GICC) (Ref. UV-INV_GIUV-172923) de la Universidad de Valencia. 
do los dosieres de los últimos cinco años (2014-2018) y se ha creado una base de datos que incluye las citas recibidas por los documentos difundidos en revistas científicas indexadas en la Web of Science y las menciones en redes sociales utilizando Altmetric.com. Posteriormente se ha realizado un análisis estadístico de la información obtenida. Los principales resultados muestran que el factor de impacto de las revistas es importante para conseguir citaciones académicas o para figurar en la conversación que se genera en Twitter. Sin embargo, la influencia del factor de impacto de la revista resulta menor a la hora de conseguir que la publicación científica obtenga popularidad en medios de comunicación. Además, las pruebas estadísticas muestran que las publicaciones científicas divulgadas a través de la UCC+i obtienen proporcionalmente más citas que las que no han sido divulgados por vía institucional.

\title{
Palabras clave
}

Noticias científicas; Comunicación institucional; Universidades; Comunicación científica; Información científica; Divulgación científica; Bibliometría; Indicadores altmétricos; Redes sociales; Universidad española; UC3M.

\begin{abstract}
Institutional offices for the communication and dissemination of science are gaining in importance at Spanish universities, and their activities can contribute to increasing the visibility and reputation of researchers. The objective of this study is to analyze the relations between the institutional communication of research results to the public and the impact and academic visibility of scientific papers. For this purpose, science communication and dissemination undertaken through press releases on $\mathrm{R}+\mathrm{D}+\mathrm{i}$ results from the Science Culture and Innovation Unit (SCIU) at Universidad Carlos III de Madrid (UC3M) were selected as a case study. This SCIU was chosen because it has published yearly reports for more than a decade on the impact in the media of its press releases. Press dossiers of the last five years available (2014-2018) were examined, and a database was created of the citations received by texts published in scientific journals indexed in the Web of Science as well as their mentions on social networks through the company Altmetric.com. After that, this information was statistically analyzed. The main results show that the journal impact index is important for obtaining academic citations or appearing in Twitter conversations. However, the influence of the journal impact factor turns out to be lower when it comes to popularizing scientific publications in the media. Furthermore, the statistical evidence points to the fact that scientific publications disseminated through the SCIU receive proportionally more citations than research that has not been disseminated through the institutional channel.
\end{abstract}

\section{Keywords}

Science news; Institutional communication; Universities; Science communication; Science popularization; Bibliometrics indicators; Altmetric indicators; Social media; Spanish university; UC3M.

\section{Introducción}

Uno de los principales objetivos y motivaciones del personal investigador en todo el mundo es cultivar una buena reputación académica, como ya han señalado varios autores clásicos (Storer, 1966; Merton, 1968; Becher, 1989) y contemporáneos (Petersen et al., 2014; Ebersole; Axt; Nosek, 2016). Sin embargo, este concepto de reputación científica no hace referencia a una sola descripción, sino que engloba varios significados, tal y como apunta un trabajo de Herman y Nicholas (2018). Después de realizar una revisión bibliográfica que incluye más de 200 publicaciones, estos autores apuntan que el concepto de reputación se configura básicamente atendiendo a dos criterios:

- productividad, tanto en términos cuantitativos como cualitativos; e

- impacto, tanto en el avance del conocimiento en su campo disciplinar como en los logros obtenidos más allá de la Academia; por ejemplo, a través de la transferencia del conocimiento a la sociedad.

Durante más de cuatro décadas, la productividad y el impacto se medían a través del análisis de las publicaciones científicas, principalmente con técnicas cienciométricas y bibliométricas, tal como afirman Callon, Courtial y Penan (1995). Sin embargo, durante esta última década, el advenimiento de la web 2.0, que ha potenciado y favorecido la comunicación y la colaboración entre distintos agentes académicos, ha modificado los parámetros para medir la producción científica del personal de investigación. Numerosas plataformas de intercambio de información como foros, blogs y aplicaciones de redes sociales (como Facebook o Twitter), no solo han tenido una buena acogida entre el público en general, sino también entre los integrantes de la comunidad científica, dando lugar a la llamada "web social académica". Este nuevo espacio de comunicación permite compartir y validar los proyectos a través de aplicaciones propias del entorno académico, como los gestores bibliográficos sociales (ej., Mendeley o Zotero), las redes profesionales, (ej. ResearchGate o ScienceOpen) y las aplicaciones de identidad digital como Publons de Web of Science $u$ Orcid (De-Filippo; Silva; Borges, 2019).

La web 2.0, que ha potenciado y favorecido la comunicación y la colaboración entre agentes académicos, ha modificado los parámetros para medir la producción científica del personal de investigación 
Una muestra del impacto de estas nuevas plataformas es el uso que le dan muchos investigadores. Por ejemplo, junto a los repositorios institucionales, ResearchGate se ha convertido en la red preferida para dar a conocer los resultados de las investigaciones, según muestran Kramer y Bosman (2016). A todo este conjunto de redes se suma el movimiento de acceso abierto a la ciencia, y los repositorios (ArXiv, e-LIS, OSF Preprints o RePEC). En este contexto, según afirman Mohammadi y Thelwall (2013), la multiplicación de canales de comunicación informales constituye un nuevo reto para el análisis de la actividad científica. Es por ello que resulta fundamental proponer nuevos modelos de abordaje y análisis de la comunicación científica que integren los canales ya consolidados junto a los nuevos. A tal efecto, los estudios bibliométricos tradicionales pueden ser ampliados y complementados con los nuevos indicadores altmétricos, que aportan una medida del interés social. El concepto de "altmetría" fue definido por Priem et al. (2010) en un post en que lo describen como la creación y estudio de nuevos indicadores (metrics) basados en la web social para analizar y caracterizar la actividad académica (scholarship).

Además de centrarse en sus resultados de investigación, los científicos deben ocuparse de su identidad profesional (marca de autor), su representación virtual y aprender a gestionarla. Por ello, según argumentan Rodríguez-Bravo y Nicholas (2018), cada vez resulta mayor la necesidad de promocionarse en las redes sociales, lo que aporta visibilidad, intercambio de conocimientos e incluso incremento de citas. Aunque la aparición de redes sociales digitales científicas ha ayudado a los investigadores a difundir sus trabajos, todavía no se ha generalizado su utilización entre el personal docente investigador de las universidades españolas (González-Díaz; Iglesias-García; Codina, 2015; Alonso-Flores; Moreno-Castro, 2018). Otro estudio apunta que menos de un 33\% del profesorado utiliza ResearchGate una vez al mes para difundir publicaciones o conseguir más citas y reputación (Campos-Freire; Rúas-Araújo, 2016). Por todo ello, resulta interesante explorar la utilización de redes sociales más generalistas, como Twitter, por parte de la comunidad científica e incorporar dicho uso como fuente de datos para los estudios cienciométricos.

Existe una corriente de opinión un tanto escéptica sobre la proactividad en Twitter porque se considera que cierto nivel de activismo distrae de la verdadera actividad investigadora. En este sentido, Hall (2014) propuso el denominado Índice Kardashian para medir la discrepancia entre el perfil de redes sociales de un científico y su registro de publicaciones, comparando números de citas y seguidores de Twitter, para tratar de cuantificar el peligro de que se puedan perder de vista las métricas críticas de valor científico ante la emergencia de las redes sociales. Hall mostró que el personal de investigación con mayor número de seguidores en Twitter era el que tenía menos citas académicas en las bases de datos internacionales, mientras que los más citados en estas bases de datos eran quienes no tenían perfil en Twitter o tenían una escasa actividad. Sin embargo, otros investigadores plantean que los científicos pueden incrementar las citaciones de sus publicaciones académicas y aumentar su impacto científico participando en discusiones en Twitter (Liang et al., 2014). En esta línea, Lamb, Gilbert y Ford (2018) han señalado que los investigadores pueden incrementar la exposición de su investigación mediante la utilización de las redes sociales y, a su vez, mejorar el desempeño académico según las métricas tradicionales de la actividad investigadora. En este contexto, parece evidente que los ciudadanos y los investigadores, en particular, juegan un papel importante en el proceso de comunicación científica en las redes sociales (Didegah; Mejlgaard; Sørensen, 2018).

En los últimos años han surgido numerosos trabajos académicos que proporcionan consejos prácticos sobre cómo afrontar la comunicación científica en las redes sociales o cómo aumentar la eficiencia y originalidad en la difusión pública en lo relativo a la investigación, desarrollo e innovación (I+D+i) (Bik; Goldstein, 2013; Dennen, 2014; Cooke et al., 2017; Pérez-Rodríguez; González-Pedraz; Alonso-Berrocal, 2018). La comunicación pública de la ciencia ha pasado a formar parte del conjunto de habilidades y conocimientos necesarios para que un científico adquiera relevancia (Chapman et al., 2015), sin embargo, resulta complicado alcanzar a las grandes audiencias que tienen los principales medios de comunicación. Para ello, la revista Nature (Kwok, 2018) recomienda que los investigadores contacten con las oficinas de prensa de sus centros de investigación. Por su parte, las instituciones públicas aspiran a una mayor transparencia y visibilidad ante la ciudadanía porque estos dos parámetros otorgan cierta imagen de honestidad (Moreno-Sardá; Rodríguez-Navas; Corcoy-Rius, 2013). Además, en el caso de las universidades, la visibilidad de las actividades de I+D+i puede mejorar su posicionamiento en determinados rankings internacionales al mejorar su reputación (Johnes, 2018; Pérez-Esparrells; López-García, 2018).

En las universidades españolas las acciones realizadas para dar visibilidad a los resultados de investigación las realizan, fundamentalmente, los gabinetes de comunicación institucional y las Unidades de Cultura Científica y de Innovación (UCC+i). Las universidades españolas tardaron décadas en integrar los gabinetes de prensa entre sus servicios y, por tanto, en ser conscientes de la importancia que tenían para sus instituciones (Alonso-Flores; Serrano-López; Moreno-Castro, 2018). Los primeros servicios de prensa aparecieron en los campus españoles en los años 70 y 80 , con la llegada de la democracia, con el objetivo de obtener cierta proyección pública a través de los medios de comunicación, un aspecto al que no se había prestado atención hasta la fecha (Moreno-Castro, 2004; Paniagua-Rojano; Gómez-Calderón; Fernández-Sande, 2012). Posteriormente, sobre todo durante los años 90, se incrementaron los gabinetes de comunicación 
en las universidades y se comenzaron a diseñar nuevas técnicas de mercadotecnia con las que difundir la imagen de la institución (Parejo-Cuéllar, 2016).

Las UCC+i se consolidaron con la Convocatoria de Ayudas para la realización de actividades de difusión y divulgación científica y tecnológica del Año de la Ciencia 2007, promovido por la Fundación Española para la Ciencia y la Tecnología (Fecyt), que incentivó la creación y fortalecimiento de este tipo de unidades en universidades y centros de investigación en España (Fecyt, 2012). Estas estructuras surgieron para responder a la necesidad de difundir lo que ocurría en materia científica y tecnológica dentro de las instituciones (Roca-Marín, 2017). Las oficinas de prensa de las universidades se habían dedicado a la comunicación institucional pero no se habían especializado en difundir y divulgar los resultados de sus grupos de investigación. La particularidad de las UCC+i es que están focalizadas en la actividad y publicaciones del personal investigador. Cerca de una treintena de universidades cuentan con UCC+i que prestan un servicio específico de comunicación dedicado a la labor de acercar la ciencia a la ciudadanía en sus diferentes formatos (Parejo-Cuéllar; Martín-Pena; Pinto-Zúñiga, 2016). Un diagnóstico reciente sobre la divulgación en las universidades españolas (CRUE, 2019), en el que participaron 62 instituciones, confirma a las UCC+i como el servicio responsable de la promoción de la cultura científica y la comunicación de la ciencia.

Los investigadores pueden incrementar la exposición de su investigación mediante la utilización de las redes sociales y, a su vez, mejorar el desempeño académico

Analizar los resultados del trabajo de comunicación pública de la ciencia que se realiza desde las universidades puede ayudar a entender el papel que desempeñan estas instituciones en este contexto informativo, ya que, tal como mencionan algunos estudios, se han convertido en uno de los pilares importantes a la hora de incrementar el nivel de cultura científica entre los jóvenes españoles (Pérez; Olvera-Lobo, 2014). Sin duda, la implementación de estas unidades ha tenido un rol fundamental en la difusión de los resultados de investigación generados en las universidades españolas por lo que resulta de sumo interés conocer su influencia en la visibilidad de estos resultados.

\section{Objetivos}

Considerando el contexto planteado, este trabajo tiene tres objetivos de investigación. En primer lugar, analizar las citas periodísticas detectadas sobre los resultados de investigación difundidos por los gabinetes de prensa y las UCC+i. En segundo lugar, estudiar las relaciones entre la difusión pública institucional de la ciencia y el impacto académico, en términos de citas recibidas y de visibilidad de las publicaciones científicas del trabajo en redes sociales, especialmente en Twitter. Y en tercer lugar, comparar las citaciones que reciben los documentos académicos que han sido difundidos mediante notas de prensa con el resto de publicaciones científicas de la institución.

Este estudio se circunscribe en una línea de investigación más amplia que trata de analizar el impacto científico y académico que puede suponer para el personal investigador y para la institución la divulgación de los trabajos de I+D+i, en términos de número de citaciones recibidas, prestigio y reputación.

\section{Metodología}

Se ha seleccionado como caso de estudio la actividad de diseminación de los resultados de las investigaciones que lleva a cabo la UCC+i de la Universidad Carlos III de Madrid (UC3M), a través de notas de prensa dirigidas a medios y redes sociales. Se escoge esta institución porque pertenece a la red de UCC+i de la Fecyt desde su origen y porque publica, desde hace más de una década, informes anuales sobre el impacto en medios de comunicación que tienen las notas de prensa que difunden. Las fuentes de información utilizadas han sido:

- Repositorio digital de la UC3M (SCI UC3M, 2019): este repositorio en acceso abierto contiene los informes (dosieres) con información sobre el impacto de las notas de prensa de investigaciones realizadas por la UCC+i de la Universidad. Una ventaja de la utilización de esta fuente es que los dosieres se han elaborado siguiendo una misma metodología, que consiste en incluir el impacto recogido por un servicio de press clipping con Acceso (ahora denominado Rebold) y complementarlo con búsquedas digitales en buscadores de Internet. https://letsrebold.com

- Base de datos bibliográfica Web of Science (WoS) de Clarivate Analytics: Se ha utilizado esta fuente multidisciplinar que ofrece información bibliográfica y bibliométrica, para obtener indicadores de impacto y visibilidad de las publicaciones científicas que han sido difundidas por la UCC+i. La información proveniente de las tres bases de datos principales ( $\mathrm{SCl}, \mathrm{SSCl}$ y $\mathrm{A \& HCl}$ ) ha sido complementada con los Journal Citation Reports (JCR).

- Almetric.com: se ha consultado esta plataforma para conocer la repercusión que alcanzan las publicaciones científicas analizadas en redes sociales. Se trata de una fuente que recoge los indicadores altmétricos asociados a los trabajos científicos. Su consulta se puede llevar a cabo de manera manual o, como se ha hecho en este caso, a través de consultas automatizadas a la API de Altmetric.com, utilizando como elemento de búsqueda el DOI (digital object identifier) de los documentos, dado que la mayor parte de los trabajos analizados contaban con este identificador.

En primer lugar, se han analizado las 198 notas de prensa difundidas desde la UCC+i de la UC3M en un periodo de 5 años: de 2014 a 2018. Estos contenidos son publicados en la web de la Universidad y se envían a medios de comunicación locales, 
las secciones de educación de medios generalistas y otros especializados en temas de ciencia, tecnología e innovación. Además, se realiza una difusión en diversas plataformas nacionales e internacionales especializadas en la comunicación de la I+D+i, como son:

- el boletín Notiweb del sistema Madri+d de la Comunidad de Madrid:

https://www.madrimasd.org/notiweb

- la Agencia de Noticias para la divulgación de la Ciencia y Tecnología del Instituto de Estudios de la Ciencia y la Tecnología (DiCYT): https://www.dicyt.com

- el Servicio de Información y Noticias Científicas (SINC) de la Fecyt: https://www.agenciasinc.es

- el servicio europeo de noticias de investigación, AlphaGalileo: https://www.alphagalileo.org

- y el servicio global online de noticias de ciencia, medicina y tecnología de la Asociación Americana para el Avance de la Ciencia, EurekAlert! (UC3M, 2019).

https://www.eurekalert.org

De las 198 notas de prensa, se han seleccionado las que versan sobre resultados de investigación: un total de 85. Con ellas se ha construido una base de datos con la siguiente información: tipo de documento (artículos de revista, presentaciones en congresos, tesis, preprints, libros, informes), referencia bibliográfica, DOI, fecha de difusión, título de la nota de prensa, nombre del investigador/a de la UC3M, número de difusiones realizadas en webs y plataformas de divulgación científicas realizadas desde la $\mathrm{UCC}+\mathrm{i}$, impacto en medios nacionales (número de menciones), el impacto en medios internacionales (número de menciones), impacto registrado en webs (número de menciones) e impacto total (menciones globales recibidas en los diferentes medios).

En segundo lugar, todas las publicaciones que dieron origen a las notas de prensa seleccionadas fueron buscadas en la WoS para obtener información sobre su impacto (número de citas recibidas) y visibilidad (cuartil de las revistas de publicación en los JCR). Para evitar sesgos, toda la información fue consultada y descargada el mismo día (15/09/2019). Los datos obtenidos fueron introducidos en la base de datos ya elaborada: título, autores, instituciones firmantes, fecha de publicación, idioma de los documentos, tipo documental, revista y cuartil, citas recibidas, categoría temática, conteo de uso (indica el número de veces que los usuarios han descargado la publicación).

En tercer lugar, se obtuvo información sobre las menciones en redes sociales de cada publicación analizada. A partir de su DOI y mediante un script desarrollado por el Laboratorio de Estudios Métricos de la Información de la UC3M, se obtuvieron de la plataforma Altmetric.com los siguientes indicadores altmétricos para cada publicación: número de menciones en Facebook, posts, Googleplus, videos, msm (mainstream media), feeds, Twitter, Wikipedia y número de lectores en Mendeley. La información obtenida fue integrada en la base de datos elaborada.

En cuarto lugar, se extrajo información general bibliométrica de la investigación de la UC3M a través de la base de datos del Observatorio de la Actividad Investigadora en la Universidad Española (IUNE, 2019), coordinado por el Laboratorio de Estudios Métricos de la Información (LEMI) de la UC3M en el marco de la Alianza 4 Universidades. Se han consultado datos como el número de documentos publicados por investigadores de la UC3M en la WoS durante el periodo analizado, el número de citas en función del cuartil de la revista y el número de citas por año, para poder establecer una comparativa con los datos referentes a los trabajos académicos que sí fueron difundidos por la UCC+i.

Una vez incluida toda la información en la base de datos se realizaron dos tipos de tests estadísticos. Por un lado, para determinar si existe diferencia significativa entre la distribución de citas de los trabajos de la UC3M y los que han sido divulgados por su UCC+i se ha escogido el test de Wilcoxon-Mann-Whitney. La elección de esta prueba no paramétrica se debe a que la distribución de las citas no sigue en ninguno de los dos casos una distribución normal, por lo que no sería apropiado aplicar otro tipo de pruebas paramétricas como la t de Student. Por otro lado, con el fin de estudiar la relación entre las citas recibidas por los trabajos y algunos indicadores altmétricos se ha utilizado el coeficiente de determinación, que indica en qué medida se ajusta la nube de puntos a una línea recta.

\section{Resultados}

Para organizar los resultados, en primer lugar se presentan las características de las notas de prensa analizadas. Seguidamente se muestran los indicadores de impacto y visibilidad poniendo en relación las variables estudiadas. 


\subsection{Características de las notas de prensa}

Entre los resultados de investigación difundidos por la UCC+i el "artículo de revista" es el tipo de documento predominante $(76 \%)$, seguido de las presentaciones a congresos ( $8 \%$ ), libros (8\%) y tesis (4\%). En promedio se han realizado 22 difusiones por documento en diversas webs institucionales y plataformas de comunicación y divulgación de la ciencia. El análisis no ha detectado diferencias entre tipos documentales.

Si se considera el impacto de la difusión institucional (la presencia de las notas de prensa en diversos medios de comunicación y webs), se aprecia que los documentos han recibido un total de 6.890 menciones ( 81,05 menciones por documento), siendo los congresos y los preprints los que han alcanzado mayor visibilidad proporcionalmente, con 197,57 y 89 citas por documento, respectivamente. Como se observa en la tabla 1, el mayor número de menciones ha sido en los medios de comunicación nacionales (23,55 menciones/doc).

Tabla 1. Menciones recibidas por las notas de prensa difundidas por la UCC+i de la UC3M. Fecha: 15-09-19

\begin{tabular}{|c|c|c|c|c|c|c|c|c|c|c|}
\hline \multirow[b]{2}{*}{$\begin{array}{l}\text { Tipo de } \\
\text { documento }\end{array}$} & \multicolumn{2}{|c|}{$\begin{array}{c}\text { Menciones en } \\
\text { difusión } \\
\text { institucional }\end{array}$} & \multicolumn{2}{|c|}{$\begin{array}{c}\text { Menciones en } \\
\text { medios nacionales }\end{array}$} & \multicolumn{2}{|c|}{$\begin{array}{c}\text { Menciones en } \\
\text { medios } \\
\text { internacionales }\end{array}$} & \multicolumn{2}{|c|}{$\begin{array}{l}\text { Menciones en } \\
\text { otras webs }\end{array}$} & \multirow{2}{*}{$\begin{array}{l}\text { Número total } \\
\text { de menciones }\end{array}$} & \multirow{2}{*}{$\begin{array}{c}\text { Número total } \\
\text { de menciones } \\
\text { por } \\
\text { documento }\end{array}$} \\
\hline & 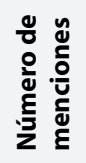 & 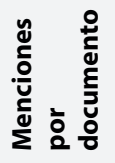 & 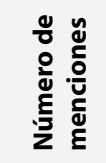 & 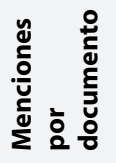 & 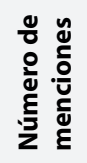 & 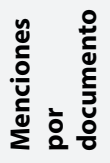 & 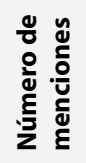 & 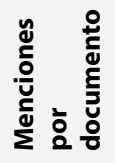 & & \\
\hline Artículo & 1.436 & 22,09 & 1.493 & 22,97 & 955 & 14,69 & 1.473 & 22,66 & 5.357 & 82,41 \\
\hline Congreso & 161 & 23 & 253 & 36,14 & 179 & 25,57 & 160 & 22,86 & 753 & 107,57 \\
\hline Informe & 25 & 25 & 20 & 20 & 5 & 5 & 16 & 16 & 66 & 66 \\
\hline Libro & 165 & 23,57 & 110 & 15,71 & 30 & 4,29 & 64 & 9,14 & 369 & 52,71 \\
\hline Preprints & 26 & 26 & 31 & 31 & 19 & 19 & 13 & 13 & 89 & 89 \\
\hline Tesis & 99 & 24,75 & 95 & 23,75 & 16 & 4 & 46 & 11,5 & 256 & 64 \\
\hline Total & 1.912 & 22,49 & 2.002 & 23,55 & 1.204 & 14,16 & 1.772 & 20,85 & 6.890 & 461,69 \\
\hline
\end{tabular}

Tabla 2. Notas de prensa con mayor impacto en medios

\begin{tabular}{|c|c|c|c|c|c|c|}
\hline $\begin{array}{l}\text { Fecha de } \\
\text { publicación }\end{array}$ & Titular de la nota de prensa & $\begin{array}{l}\text { Impacto en } \\
\text { medios }\end{array}$ & $\begin{array}{l}\text { Tipo de } \\
\text { documento }\end{array}$ & $\begin{array}{l}\text { Cuartil de } \\
\text { la revista }\end{array}$ & $\begin{array}{l}\text { Citas en } \\
\text { WoS }\end{array}$ & $\begin{array}{l}\text { Menciones } \\
\text { en Twitter }\end{array}$ \\
\hline 23/01/2017 & $\begin{array}{l}\text { Científicos españoles crean una bioimpresora 3D de } \\
\text { piel humana }\end{array}$ & 616 & Artículo & Q1 & 53 & 182 \\
\hline $11 / 11 / 2014$ & $\begin{array}{l}\text { Los robots humanoides más importantes del mundo } \\
\text { se citan en Madrid la próxima semana }\end{array}$ & 265 & Congreso & - & - & - \\
\hline $14 / 11 / 2017$ & $\begin{array}{l}\text { ¿Por qué el agua caliente puede congelarse antes que } \\
\text { el agua fría? }\end{array}$ & 200 & Artículo & Q1 & 6 & - \\
\hline 20/04/2015 & Desarrollan un terapeuta robótico para niños & 172 & Artículo & Q4 & 0 & - \\
\hline $18 / 03 / 2015$ & $\begin{array}{l}\text { Una investigación analiza la rivalidad histórica del } \\
\text { Real Madrid y el FC Barcelona }\end{array}$ & 170 & Artículo & - & 3 & - \\
\hline $15 / 01 / 2016$ & $\begin{array}{l}\text { Realidad virtual para la rehabilitación motora del } \\
\text { hombro }\end{array}$ & 166 & $\begin{array}{l}\text { Actas de } \\
\text { congreso }\end{array}$ & - & 16 & - \\
\hline $15 / 09 / 2016$ & $\begin{array}{l}\text { Un estudio UC3M sobre el comportamiento humano } \\
\text { distingue cuatro tipos básicos de personalidad }\end{array}$ & 150 & Artículo & Q1 & 19 & 271 \\
\hline $11 / 03 / 2016$ & $\begin{array}{l}\text { Redes sociales para evaluar daños provocados por } \\
\text { desastres naturales }\end{array}$ & 145 & Artículo & Q1 & 80 & 370 \\
\hline $23 / 07 / 2018$ & $\begin{array}{l}\text { Un estudio científico caracteriza nuestros círculos de } \\
\text { amistad }\end{array}$ & 134 & Artículo & Q1 & 1 & 142 \\
\hline $16 / 03 / 2015$ & $\begin{array}{l}\text { Nuevo sistema para detectar efectos adversos de los } \\
\text { medicamentos usando redes sociales }\end{array}$ & 130 & Artículo & Q2 & 23 & - \\
\hline $26 / 04 / 2018$ & $\begin{array}{l}\text { Cuando hace buen tiempo, somos más felices en las } \\
\text { redes sociales }\end{array}$ & 119 & Artículo & Q1 & 9 & 395 \\
\hline $10 / 11 / 2014$ & $\begin{array}{l}\text { Solo el } 6 \% \text { de las personas trabajan en lo que soñaron } \\
\text { de niños }\end{array}$ & 111 & Artículo & Q1 & 7 & 10 \\
\hline $20 / 06 / 2018$ & $\begin{array}{l}\text { Desarrollan una herramienta que muestra la brecha } \\
\text { de género en Facebook }\end{array}$ & 108 & Artículo & Q1 & 2 & 143 \\
\hline $31 / 07 / 2015$ & $\begin{array}{l}\text { La mitad de las noticias con éxito en Twitter no apare- } \\
\text { cen en los medios de comunicación tradicionales }\end{array}$ & 103 & Artículo & Q1 & 5 & 44 \\
\hline $25 / 04 / 2016$ & $\begin{array}{l}\text { Los portales de vídeo en internet no controlan bien } \\
\text { las visitas }\end{array}$ & 101 & $\begin{array}{l}\text { Actas de } \\
\text { congreso }\end{array}$ & - & 5 & - \\
\hline
\end{tabular}


De las 85 notas de prensa analizadas, 69 contaban con DOI (81\%) y de ellas, 60 fueron sobre artículos indexados en revistas de la WoS. Las principales revistas de publicación fueron: PloS one y Physical review letters. De las 60 publicaciones, $40(66,6 \%)$ han contado con menciones en redes sociales, siendo los posts, los msm y Twitter las fuentes más frecuentes.

Tras analizar toda la información de las 85 notas de prensa, se ha comprobado cuáles han sido las 15 que han logrado mayor visibilidad en medios de comunicación (se han seleccionado las que han superado el centenar de menciones en webs y medios de comunicación). Se muestran en la tabla 2 con su fecha de publicación, titular y el total de menciones que ha recibido en medios de comunicación. Se presenta también el tipo de documento, el cuartil de la revista en el caso de los artículos, las citas recibidas en WoS y las menciones en redes sociales.

Estas 15 notas de prensa provienen de 12 publicaciones académicas en revistas científicas y de 3 en congresos científicos. En el caso de las revistas científicas, 9 son del primer cuartil (Q1), según se ha comprobado en los JCR; es decir, corresponden a las revistas con mayor índice de impacto de sus respectivas áreas temáticas. Otras 2 son noticias a partir de artículos publicados en revistas Q2 y Q4, respectivamente. Por otra parte, al analizar los trabajos divulgados que han tenido un mayor número de citas académicas en WoS (ver tabla 3), se comprueba que 14 de los 15 artículos más citados corresponden a revistas Q1 y la otra a Q2. Y en el caso de los 15 trabajos que han tenido más éxito en redes sociales se ha podido comprobar que todos han sido publicados en revistas del primer cuartil.

Cuando se analizan las 15 notas de prensa que han tenido un mayor impacto académico según el número de citas recibidas en el periodo analizado (ver tabla 3), se comprueba que solo 4 aparecen entre las más populares en medios de comunicación. En cambio, cuando se examinan las 15 notas de prensa que han tenido un mayor número de menciones en Twitter, más de la mitad (8) figuran también entre las que más citaciones reciben, es decir, figuran también en la tabla 2. Y algo similar ocurre, aunque en menor proporción, entre las notas de prensa con más éxito en medios de comunicación, que coinciden en 7 casos con las más populares en Twitter.

Tabla 3. Notas de prensa con mayor impacto académico

\begin{tabular}{|c|c|c|c|c|c|c|}
\hline $\begin{array}{l}\text { Fecha de } \\
\text { publicación }\end{array}$ & Título de la nota de prensa & Citas WoS & $\begin{array}{l}\text { Tipo de } \\
\text { documento }\end{array}$ & $\begin{array}{l}\text { Cuartil de } \\
\text { la revista }\end{array}$ & $\begin{array}{l}\text { Impactos } \\
\text { en medios }\end{array}$ & $\begin{array}{l}\text { Menciones } \\
\text { en Twitter }\end{array}$ \\
\hline $11 / 03 / 2016$ & $\begin{array}{l}\text { Redes sociales para evaluar daños provocados por } \\
\text { desastres naturales }\end{array}$ & 80 & Artículo & Q1 & 145 & 370 \\
\hline $01 / 02 / 2018$ & $\begin{array}{l}\text { Un estudio analiza el potencial y los retos de los } \\
\text { metamateriales mecánicos flexibles }\end{array}$ & 77 & Artículo & Q1 & 44 & 46 \\
\hline 09/09/2014 & $\begin{array}{l}\text { Un sistema facilita la identificación del malware de } \\
\text { los smartphones }\end{array}$ & 75 & Artículo & Q1 & 58 & 3 \\
\hline $21 / 12 / 2016$ & $\begin{array}{l}\text { El cerebro experimenta cambios adaptativos durante } \\
\text { el embarazo }\end{array}$ & 73 & Artículo & Q1 & 17 & 1.376 \\
\hline $23 / 01 / 2017$ & $\begin{array}{l}\text { Científicos españoles crean una bioimpresora 3D de } \\
\text { piel humana }\end{array}$ & 53 & Artículo & Q1 & 616 & 182 \\
\hline $13 / 12 / 2016$ & $\begin{array}{l}\text { Las empresas prudentes en su contabilidad obtienen } \\
\text { más financiación }\end{array}$ & 48 & Artículo & Q1 & 17 & - \\
\hline 05/05/2014 & Un coche inteligente detecta peatones de noche & 41 & Artículo & Q1 & 74 & - \\
\hline $28 / 04 / 2014$ & $\begin{array}{l}\text { Un sistema detecta con dos meses de antelación las } \\
\text { tendencias mundiales en redes sociales }\end{array}$ & 38 & Artículo & Q1 & 88 & 204 \\
\hline $23 / 01 / 2018$ & $\begin{array}{l}\text { Crean una nueva base de datos de videos sobre } \\
\text { seguimiento celular útil en la lucha contra el cáncer }\end{array}$ & 36 & Artículo & Q2 & 66 & 117 \\
\hline $16 / 03 / 2015$ & $\begin{array}{l}\text { Nuevo sistema para detectar efectos adversos de los } \\
\text { medicamentos usando redes sociales }\end{array}$ & 23 & Artículo & Q1 & 130 & - \\
\hline $27 / 01 / 2014$ & $\begin{array}{l}\text { Nuevos diagnósticos biomédicos por imagen en 3D } \\
\text { personalizables }\end{array}$ & 22 & Artículo & Q1 & 72 & - \\
\hline $26 / 05 / 2014$ & Los intermediarios aumentan la corrupción & 20 & Artículo & Q1 & 78 & 9 \\
\hline $28 / 05 / 2014$ & $\begin{array}{l}\text { La cooperación se aprende con la práctica, según un } \\
\text { modelo matemático }\end{array}$ & 20 & Artículo & Q1 & 34 & 10 \\
\hline $15 / 09 / 2016$ & $\begin{array}{l}\text { Un estudio UC3M sobre el comportamiento humano } \\
\text { distingue cuatro tipos básicos de personalidad }\end{array}$ & 19 & Artículo & Q1 & 86 & 271 \\
\hline 09/07/2014 & $\begin{array}{l}\text { La cooperación entre humanos, una cuestión de } \\
\text { edad, según una investigación UC3M }\end{array}$ & 19 & Artículo & Q1 & 150 & 56 \\
\hline
\end{tabular}

\subsection{Visibilidad e impacto de las publicaciones difundidas por la UCC+i}

En cuanto al impacto académico de las publicaciones difundidas por la UCC+i (e indexadas en WoS), han alcanzado un promedio de 16 citas/doc. Estos valores se han comparado con los alcanzados por el total de documentos publicados por la UC3M (8,81 citas/doc) poniendo de manifiesto que las publicaciones difundidas por la UCC+i casi duplican el impac- 
to promedio de los documentos de la universidad. Dado que suele existir una relación positiva entre la visibilidad y el impacto académico (las publicaciones en revistas de mejores cuartiles -Q1 y Q2- obtienen más citas), se han calculado las citas recibidas para el conjunto de publicaciones de cada cuartil. Como se muestra en la tabla 4, los valores de impacto (citas/doc) en Q1 y Q2 son mucho mayores en el caso de las publicaciones difundidas institucionalmente (*el total de documentos en los $J C R$ y de citas es menor que el sumatorio por cuartiles porque un mismo documento puede estar en más de un cuartil en función de la posición que alcance en las distintas disciplinas en que se clasifique la revista en la que ha sido publicado).

Tabla 4. Comparación del promedio de citas de las publicaciones científicas generales de la UC3M y las difundidas por la UCC+i en función del cuartil (Q) de la revista de publicación

\begin{tabular}{|c|c|c|c|c|c|c|c|c|c|c|c|c|c|c|c|}
\hline & \multicolumn{5}{|c|}{ Número de documentos } & \multicolumn{5}{|c|}{ Número de citas } & \multicolumn{5}{|c|}{ Citas por documento } \\
\hline & Q1 & Q2 & Q3 & Q4 & $\begin{array}{c}\text { Total docs. } \\
\text { JCR }\end{array}$ & Q1 & Q2 & Q3 & Q4 & $\begin{array}{l}\text { Total } \\
\text { citas * }\end{array}$ & Q1 & Q2 & Q3 & Q4 & $\begin{array}{l}\text { Promedio } \\
\text { de citas/ } \\
\text { doc. }\end{array}$ \\
\hline Total UC3M & 2.364 & 1.731 & 990 & 549 & 4.564 & 23.521 & 10.165 & 3.963 & 1.522 & 40.190 & 9,95 & 5,87 & 4 & 2,77 & 8,81 \\
\hline $\begin{array}{l}\text { Documentos con } \\
\text { difusión institu- } \\
\text { cional }\end{array}$ & 39 & 3 & 6 & 2 & 50 & 797 & 28 & 22 & 849 & 849 & 20,44 & 9,33 & 3,67 & 1 & 16 \\
\hline
\end{tabular}

Aplicando un test de Wilcoxon-Mann-Whitney para ambas distribuciones se obtiene una diferencia estadísticamente significativa entre ambas, con un p-valor de 0,001435 . Frente a estos resultados se podría justificar que las publicaciones científicas difundidas a través de la UCC+i de la UC3M son, en su mayoría, artículos de revistas publicados en el primer cuartil de los $J C R$, por lo que podría considerarse que existe algún tipo de sesgo. Para evitarlo, se ha repetido el test estadístico limitando ambas distribuciones a los artículos del primer cuartil y se ha identificado que, en este caso, la media de citas de los artículos difundidos por la UCC+i es de 20,43 y la de los trabajos publicados por autores de la UC3M en la WoS (primer cuartil) es de 9,94, obteniendo en el test de Wilcoxon-Mann-Whitney un $\mathrm{p}$-valor de nuevo inferior a 0,05; en este caso, 0,0001302. Por lo que, tal y como muestran las pruebas estadísticas, los trabajos difundidos a través de la UCC+i obtienen proporcionalmente más citas que los que no lo hacen, mostrando además una distribución mucho más uniforme (ver figura 1), con una proporción de trabajos no citados (6,89\%) menor que la del total de trabajos publicados por el personal de investigación de la UC3M (19,51\%).

Tal como se puede observar en las figuras 2 y 3 , al correlacionar el número de citas recibidas por las publicaciones científicas de la UC3M con el impacto total de la difusión (las menciones de las notas de prensa en diversos medios de comunicación y webs) y con el número de menciones en Twitter, se obtienen coeficientes de determinación muy bajos (0,03 y 0,001 respectivamente). Por las imágenes que muestran las figuras 2 y 3 se puede inferir que no existe ningún tipo de relación lineal entre estas variables. Es decir, un impacto muy elevado en los medios de comunicación y en Twitter, no se correlaciona con publicaciones muy citadas académicamente. En las figuras se puede observar algún dato atípico, causado sobre todo por un documento (el que corresponde a la nota de prensa "Científicos españoles crean una bioimpresora 3D de piel humana", que recibió 53 citas y tuvo 616 menciones en la difusión en webs y medios), pero se ha decidido 
mantenerlo en el análisis estadístico para realizar un estudio completo y riguroso de toda la muestra. No obstante, al quitar este documento destacado del análisis, la correlación también es inexistente e incluso resulta inversa.

\section{Discusión y conclusiones}

Esta investigación se basa en un estudio de caso que puede ser representativo del trabajo de comunicación y transferencia del conocimiento que se realiza en las universidades españolas, al tratarse de una de las UCC+i más longevas a nivel nacional y encontrarse circunscrita en el gabinete de comunicación institucional de la Universidad Carlos III de Madrid (UC3M). Además, el disponer de una fuente de información primaria como los dosieres con datos sobre el impacto en webs y medios de comunicación obtenido por las notas de prensa que elabora esta $U C C+i$ resulta fundamental para abordar este estudio. Según Casino (2018), la prensa es el principal productor de noticias y ofrece un producto más consolidado y con un filtro profesional y, por tanto, muy positivo para la transferencia del conocimiento. No se trata, en todo caso, de enfrentar "cita periodística" frente a "cita científica", sino de que ambas puedan sumar en la visibilización de los avances de la ciencia en la sociedad.

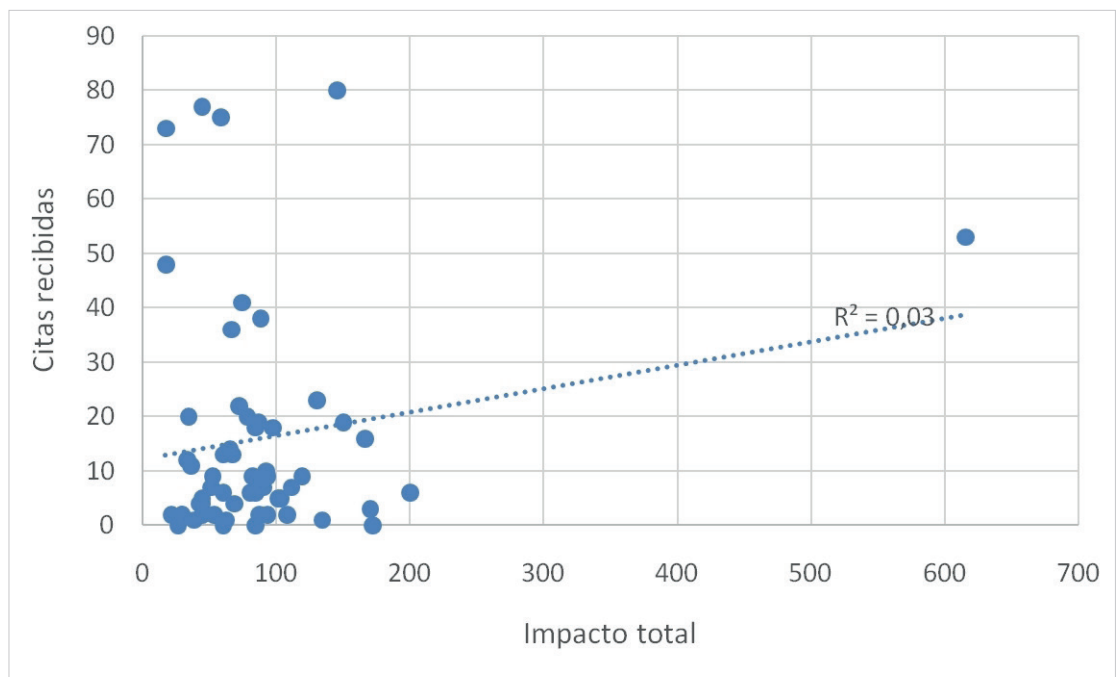

Figura 2. Impacto de la difusión institucional total vs. citas recibidas

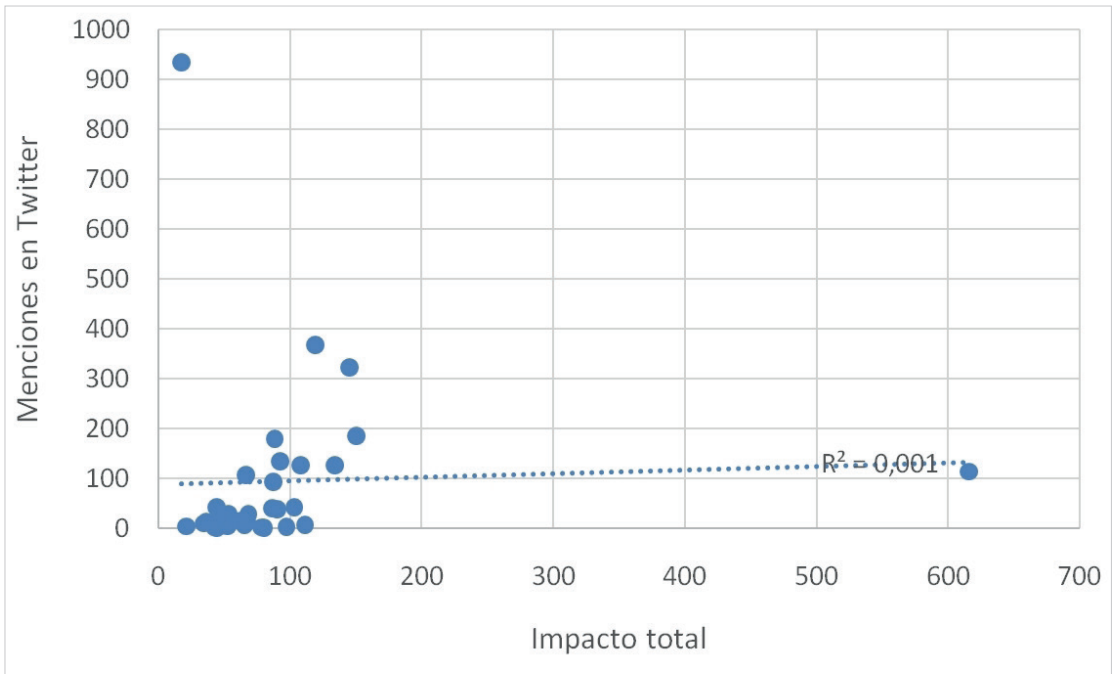

Figura 3. Impacto de la difusión institucional total vs. menciones en Twitter

Según el tipo de documento científico, las notas prensa que más éxito han tenido en medios han sido las correspondientes a informaciones sobre congresos y preprints, con un rendimiento muy superior al de las notas de prensa sobre artículos científicos. Esto puede ocurrir porque en determinados congresos se presentan prototipos y artilugios científicos que despiertan el interés de los medios por su espectacularidad, como ocurre en el caso de los robots en los congresos de esta área de investigación (por ejemplo, ver la segunda y sexta noticia de la tabla 2). En relación con los preprints, hace décadas, como ya señaló Russell (2001), se convirtieron en el principal método para informar sobre nuevos hallazgos para investigadores en campos como las matemáticas, la física o la informática, de manera que resulta habitual que sus contenidos encuentren eco en medios de comunicación. El papel transformador de los preprints en la aceleración de la comunicación académica ha sido analizado en informes recientes (Chiarelli et al., 2019), que señalan entre sus beneficios la rápida difusión y el aumento del acceso a los resultados de la investigación.

Entre los hallazgos más destacados del presente estudio se encuentra la mayor visibilidad mediática obtenida por las publicaciones científicas que han sido objeto de difusión institucional a través de la UCC+i, alcanzando un mayor número de citas académicas (un promedio de 18,98 citas, frente a las 8,81 citas por documento que consigue de media el total de las publicaciones del personal de investigación de la UC3M) y con una proporción de trabajos no citados menor (6,89\%) que la media general de la Universidad (19,51\%). Sin embargo, las pruebas estadísticas apuntan que un impacto muy elevado en los medios de comunicación y en Twitter no se correlaciona con trabajos muy citados académicamente. Estos resultados no invalidan el hecho de que los trabajos difundidos por la UCC+i hayan obtenido proporcionalmente más citas, sino que indican que estos indicadores responden a otro tipo de intereses $y$,

La comunicación pública de la ciencia ha pasado a formar parte del conjunto de habilidades y conocimientos necesarios para que un científico adquiera relevancia 
por tanto, no actúan como predictores del número de citas alcanzado por las publicaciones científicas.

Estos resultados coinciden con los de otra investigación (González-Pedraz et al., 2018), que sugiere que las UCC+i están favoreciendo la visibilidad de la ciencia española y que los medios digitales con escasos recursos dependen informativamente de ellas. Se puede determinar, por tanto, que las UCC+i de las universidades juegan un papel importante en la difusión y divulgación de los trabajos científicos producidos en la propia institución. La correlación entre la publicación de una investigación en medios de comunicación y el incremento en las citaciones científicas que se ha hallado en el marco de este estudio, ya fueron señaladas por Phillips et al. (1991), que identificaron que los artículos científicos que se habían publicado en The New York Times habían sido citados un $70 \%$ más que los que no aparecieron en este diario de referencia. Otros trabajos posteriores han encontrado una correlación positiva entre artículos publicados en revistas científicas y, paralelamente, en revistas de divulgación, de diversas disciplinas, en 13 países (Bentley; Kyvik, 2011).

A partir de los datos obtenidos en la presente investigación, se verifica que el alto nivel de citas de un trabajo en bases de datos académicas no se corresponde con los criterios que siguen los medios de comunicación para seleccionar contenidos. De hecho, un $22 \%$ de las noticias (basadas en investigaciones académicas), que tienen más éxito en los medios de comunicación, no se corresponden con trabajos publicados en revistas del primer cuartil. Sin embargo, esto no sucede en el caso de Twitter, donde se observa que los 15 trabajos con mayor éxito en esta red social se corresponden en su totalidad con estudios publicados en revistas Q1. Este resultado coincide con otros trabajos (De-Filippo; Serrano-López, 2018), que apuntan que uno de los factores más significativos para el impacto en las redes sociales es el prestigio y la reputación de la revista en la que se publica. En este sentido, podría resultar interesante analizar el papel que desempeñan las propias revistas científicas como agentes dinamizadores y promotores en redes sociales, dado que las más influyentes suelen contar con gabinetes de prensa que les ayudan a gestionar su comunicación mediática, sus relaciones públicas y su visibilidad, como han señalado varios autores (Elías-Pérez, 2008; Franzen, 2012). Además, puede resultar interesante analizar el papel que desempeñan los propios investigadores en relación con la presencia de sus trabajos en redes sociales, dado que la gran mayoría considera Twitter como un instrumento útil para mejorar la comunicación científica (Alonso-Flores; Moreno-Castro; Serrano-López, 2019).

Los resultados de este estudio permiten conocer un poco mejor las interacciones que surgen entre los gabinetes de comunicación institucional y las UCC+i frente a los medios de comunicación. No obstante, conviene señalar algunas limitaciones de este trabajo, tanto por el tamaño de la muestra como por tratarse de un estudio de caso que puede no ser generalizable ni extrapolarse a otras instituciones. Por ello, podría llevarse a cabo un estudio con resultados de otras universidades e instituciones científicas que realicen difusión institucional de su I+D+i, aunque resulta complicado encontrar una base de datos abierta, pública y con una periodicidad amplia del impacto en webs y medios de comunicación de la difusión institucional del I+D+i como la que se ha utilizado en este estudio. Este hecho, que aporta un valor singular a la investigación, también apunta hacia otra limitación, porque los datos del impacto en prensa no son reproducibles, puesto que se basan en datos de un servicio de press clipping complementado con búsquedas digitales personales, lo que aporta un componente azaroso. Por ello, en otros estudios se podría explorar la utilización exclusiva de bases de datos de prensa, como pueden ser Factiva, LexisNexis o MyNews, por ejemplo. Además, en otros futuros trabajos se podría profundizar en el rol que desempeñan las revistas científicas y los propios autores a la hora de dinamizar y difundir los trabajos en las redes sociales.
Los resultados de este estudio permiten conocer un poco mejor las interacciones que surgen entre los gabinetes de comunicación institucional y las UCC+i frente a los medios de comunicación

\section{Referencias}

Alonso-Flores, Francisco-Javier; Moreno-Castro, Carolina (2018). “Does science communication enhance researcher impact? A survey among scientists at Spanish universities". Journal of education and social policy, v. 5, n. 2, pp. 34-44. https://doi.org/10.30845/jesp.v5n2p5

Alonso-Flores, Francisco-Javier; Moreno-Castro, Carolina; Serrano-López, Antonio-Eleazar (2019). “Edad, género y estatus profesional de los investigadores como indicadores de la percepción de Twitter en la difusión de la ciencia". Perspectivas de la comunicación, v. 12, n. 1, pp. 157-184. https://doi.org/10.4067/S0718-48672019000100157

Alonso-Flores, Francisco-Javier; Serrano-López, Antonio-Eleazar; Moreno-Castro, Carolina (2018). “¿Cómo perciben los investigadores españoles la publicación de noticias sobre los resultados de sus actividades de I+D+i?". InMediaciones de la comunicación, v. 13, n. 2, pp. 115-140.

https://doi.org/10.18861/ic.2018.13.2.2870 
Becher, Toby (1989). Academic tribes and territories: Intellectual enquiry and the cultures of disciplines. Open university press. Society for research into higher education. ISBN: 9780335092215

Bentley, Peter; Kyvik, Svein (2011). "Academic staff and public communication: a survey of popular science publishing across 13 countries". Public understanding of science, v. 20, n. 1, pp. 48-63.

https://doi.org/10.1177/0963662510384461

Bik, Holly M.; Goldstein, Miriam C. (2013): "An introduction to social media for scientists". PLoS biology, v. 11, n. 4, e1001535.

https://doi.org/10.1371/journal.pbio.1001535

Callon, Michel; Courtial, Jean-Pierre; Penan, Hervé (1995). Cienciometría: la medición de la actividad científica: de la bibliometría a la vigilancia tecnológica. Gijón: Trea. ISBN: 8487733948

Campos-Freire, Francisco; Rúas-Araújo, José (2016). "Uso de las redes sociales digitales profesionales y científicas: el caso de las 3 universidades gallegas". El profesional de la información, v. 25, n. 3, pp. 431-440.

https://doi.org/10.3145/epi.2016.may.13

Casino, Gonzalo (2018). "Cita periodística: impacto de las revistas y los artículos científicos en la prensa generalista". El profesional de la información, v. 27, n. 3, pp. 692-697.

https://doi.org/10.3145/epi.2018.may.22

Chapman, Jacqueline-Marie; Algera, Dirk; Dick, M.; Hawkins, Emily E.; Lawrence, Michael J.; Lennox, Robert-James; Rous, Andrew; Souliere, Christopher M.; Stemberger, Holly L. J.; Struthers, Daniel P.; Ward, Taylor D.; Zolderdo, Aaron J.; Cooke, Steven J. (2015). "Being relevant: practical guidance for early career researchers interested in solving conservation problems". Global ecology and conservation, v. 4, pp. 334-348.

https://doi.org/10.1016/j.gecco.2015.07.013

Chiarelli, Andrea; Johnson, Rob; Richens, Emma; Pinfield, Stephen (2019). “Accelerating scholarly communication: the transformative role of preprints". Bristol: Knowledge exchange office.

https://doi.org/10.5281/zenodo.3357727

Cooke, Steven J.; Gallagher, Austin J.; Sopinka, Natalie M.; Nguyen, Vivian M.; Skubel, Rachel A.; Hammerschlag, Neil; Boon, Sarah; Young, Nathan; Danylchuk, Andy J. (2017). "Considerations for effective science communication". Facets, v. 2, pp. 233-248.

https://doi.org/10.1139/facets-2016-0055

Crue (Conferencia de Rectores de las Universidades Españolas) (2019). Diagnóstico sobre la comunicación de la ciencia y la divulgación en las universidades españolas. Red Divulga de CRUE. XXVII Jornadas de investigación de las universidades españolas, 13-15 de noviembre de 2019, Córdoba, España.

http://www.uco.es/jornadascrueinvestigacion/images/pdf/JornadasCRUE-RedDIVULGA.pdf

De-Filippo, Daniela; Serrano-López, Antonio-Eleazar (2018). "From academia to citizenry. Study of the flow of scientific information from projects to scientific journals and social media in the field of 'energy saving'". Journal of cleaner production, v. 199, pp. 248-256.

https://doi.org/10.1016/j.jclepro.2018.07.177

De-Filippo, Daniela; Silva, Paulo; Borges, Maria-Manuel (2019). “Caracterización de las publicaciones de España y Portugal sobre open science y análisis de su presencia en las redes sociales". Revista española de documentación científica, v. 42 , n. 2, e235.

https://doi.org/10.3989/redc.2019.2.1580

Dennen, Vanessa P. (2014): "Becoming a blogger: trajectories, norms, and activities in a community of practice". Computers in human behavior, v. 36, pp. 350-358.

https://doi.org/10.1016/j.chb.2014.03.028

Didegah, Fereshteh; Mejlgaard, Niels; Sørensen, Mads P. (2018). "Investigating the quality of interactions and public engagement around scientific papers on Twitter". Journal of informetrics, v. 12, n. 3, pp. 960-971.

https://doi.org/10.1016/j.joi.2018.08.002

Ebersole, Charles R.; Axt, Jordan R.; Nosek, Brian A. (2016). "Scientists' reputations are based on getting it right, not being right". PLoS biology, v. 14, n. 5, e1002460.

https://doi.org/10.1371/journal.pbio.1002460

Elías-Pérez, Carlos-José (2008). "Science and scientists turned into news and media stars because of PR strategies of scientific journals: Studying its consequences in the present scientific behaviour". Journal of science communication, $\mathrm{v}$. 7, n. 3.

https://jcom.sissa.it/sites/default/files/documents/Jcom0703\%282008\%29L01_es.pdf 
Fecyt (2012). Libro blanco de las Unidades de Cultura Científica y de la Innovación, UCC+i. Fundación Española para la Ciencia y la Tecnología.

https://www.fecyt.es/es/publicacion/libro-blanco-de-las-unidades-de-cultura-cientifica-y-de-la-innovacion-ucci

Franzen, Martina (2012). "Making science news: The press relations of scientific journals and implications for scholarly communication". In: Rödder S.; Franzen M.; Weingart P. (eds.) The sciences' media connection - Public communication and its repercussions. Sociology of the sciences yearbook, v. 28. Springer, Dordrecht. ISBN: 9789400720848 https://doi.org/10.1007/978-94-007-2085-5_17

González-Díaz, Cristina; Iglesias-García, Mar; Codina, Lluís (2015). “Presencia de las universidades españolas en las redes sociales digitales científicas: caso de los estudios de comunicación". El profesional de la información, v. 24, n. 5, pp. 640-647.

https://doi.org/10.3145/epi.2015.sep.12

González-Pedraz, Cristina; Pérez-Rodríguez, Ana-Victoria; Campos-Domínguez, Eva; Quintanilla, José-Ángel (2018). "Estudio de caso sobre las Unidades de Cultura Científica (UCC+i) españolas en la prensa digital". Doxa comunicación, v. 26, pp. 169-189.

https://doi.org/10.31921/doxacom.n26a8

Hall, Neil (2014). "The Kardashian index: a measure of discrepant social media profile for scientists". Genome biology, v. 15, n. 424.

https://doi.org/10.1186/s13059-014-0424-0

Herman, Eti; Nicholas, David (2019). "Scholarly reputation building in the digital age: An activity-specific approach. Review article". El profesional de la información, v. 28, n. 1, e280102.

https://doi.org/10.3145/epi.2019.ene.02

IUNE (2019). Observatorio de la Actividad Investigadora en la Universidad Española. http://www.iune.es/es_ES

Johnes, Jill (2018). “University rankings: What do they really show?”. Scientometrics, v. 115, n. 1. pp. 585-606. https://doi.org/10.1007/s11192-018-2666-1

Kramer, Bianca; Bosman, Jeroen (2016). Innovations in scholarly communication. https://101innovations.wordpress.com

Kwok, Roberta (2018). "Press ahead". Nature, v. 560, (7717), pp. 271-273. https://cpb-us-e1.wpmucdn.com/blogs.cornell.edu/dist/1/1221/files/2018/08/Kwok.2018.working-with-press-office-1dmfj8u.pdf

Lamb, Clayton T.; Gilbert, Sophie-Louise; Ford, Adam T. (2018). "Tweet success? Scientific communication correlates with increased citations in ecology and conservation". PeerJ, v. 6, e4564.

https://doi.org/10.7717/peerj.4564

Liang, Xuan; Su, Leona-Yi-Fan; Yeo, Sara K.; Scheufele, Dietram A.; Brossard, Dominique; Xenos, Michael; Nealey, Paul; Corley, Elizabeth A. (2014). "Building buzz: (Scientists) communicating science in new media environments". Journalism \& mass communication quarterly, v. 91, n. 4, pp. 772-791.

https://doi.org/10.1177/1077699014550092

López-Pérez, Lourdes; Olvera-Lobo, María Dolores (2014). "Science communication 2.0: The situation of Spain through its public universities and the most widely-circulated online newspapers". Information resources management journal, v. 27 , n. 3, pp. $42-58$.

https://doi.org/10.4018/irmj.2014070104

Merton, Robert K. (1968). "The Matthew effect in science". Science, v. 159, n. 3810, pp. 56-63.

https://doi.org/10.1126/science.159.3810.56

Mohammadi, Ehsan; Thelwall, Mike (2013). "Assessing the Mendeley readership of social sciences and humanities research". In: Proceedings of ISSI 2011: The $13^{\text {th }}$ conference of the International Society for Scientometrics and Informetrics, v. $1-2$.

https://publons.com/publon/21529614

Moreno-Castro, Carolina (2004). “La información científico-técnica”. En: Fernández del Moral, Javier (coordinador). Periodismo especializado. Madrid: Ariel, pp. 239-262. ISBN: 9788434413023

Moreno-Sardá, Amparo; Rodríguez-Navas, Pedro-Molina; Corcoy-Rius, Marta (2013). "La información de las administraciones públicas locales. Las webs de los ayuntamientos de Cataluña". Revista latina de comunicación social, v. 68, pp. $21-27$. https://doi.org/10.4185/RLCS-2013-987 
Nicholas, David; Herman, Eti; Jamali, Hamid; Rodríguez-Bravo, Blanca; Boukacem-Zeghmouri, Chérifa; Dobrowolski, Tom; Pouchot, Stephanie (2015). "New ways of building, showcasing, and measuring scholarly reputation". Learned publishing, v. 28, n. 3, pp. 169-183.

https://doi.org/10.1087/20150303

Olvera-Lobo, María-Dolores; López-Pérez, Lourdes (2014). "Science communication 2.0: The situation of Spain through its public universities and the most widely-circulated online newspapers". Information resources management journal, v. 27, n. 3, pp. $42-58$.

https://doi.org/10.4018/irmj.2014070104

Paniagua-Rojano, Francisco-Javier; Gómez-Calderón, Bernardo J.; Fernández-Sande, Manuel (2012). "La incorporación de los departamentos de comunicación de las universidades españolas al entorno digital. Un análisis cuantitativo". Estudios sobre el mensaje periodístico, v. 18, pp. 691-701.

https://doi.org/10.5209/rev_ESMP.2012.v18.40948

Parejo-Cuéllar, Macarena (2016). Los gabinetes de comunicación de las Universidades españolas: propuesta de modelo y análisis de las salas de prensa virtuales universitarias. Tesis doctoral en la Universidad de Extremadura. http://hdl.handle.net/10662/4172

Parejo-Cuéllar, Macarena; Martín-Pena, Daniel; Pinto-Zúñiga, Regina (2016). "El nuevo rol de las universidades en la comunicación científica". En: Actas del I Congreso internacional comunicación y pensamiento. Comunicracia y desarrollo social, pp. 523-539. Sevilla: Egregius. ISBN: 9788494524325

https://idus.us.es/xmlui/handle/11441/50594

Pérez-Esparrells, Carmen; López-García, Ana M. (2018). “Los rankings de las instituciones de educación superior: una revisión del panorama internacional”. Calidad en la educación, v. 30, pp. 328-343.

https://doi.org/10.31619/caledu.n30.184

Pérez-Rodríguez, Ana-Victoria; González-Pedraz, Cristina; Alonso-Berrocal, José-Luis (2018). "Twitter como herramienta de comunicación científica en España. Principales agentes y redes de comunicación". Communication papers: media literacy and gender studies, v. 7, n. 13, pp. 95-112.

https://doi.org/10.33115/udg_bib/cp.v7i13.21986

Petersen, Alexander-Michael; Fortunato, Santo; Pan, Rag K.; Kaski, Kimmo; Penner, Orion; Rungi, Armando; Riccaboni, Massimo; Stanley, H. Eugene; Pammolli, Fabio (2014). "Reputation and impact in academic careers". PNAS, v. 43, pp. 15316-15321.

https://doi.org/10.1073/pnas.1323111111

Phillips, David P.; Kanter, Elliot J.; Bednarczyk, Bridget; Tastad, Patricia L. (1991). "Importance of the lay press in the transmission of medical knowledge to the scientific community". The New England journal of medicine, v. 325, n. 16, pp. 1180-1183.

https://doi.org/10.1056/NEJM199110173251620

Priem, Jason; Taraborelli, Dario; Groth, Paul; Neylon, Cameron (2010). Altmetrics: A manifesto, 26 October. http://altmetrics.org/manifesto

Roca-Marín, Delfina (2017). La divulgación científica en la universidad desde su contextualización histórica: estudio de caso y propuesta de un modelo de divulgación para la Universidad de Murcia. Tesis doctoral en la Universidad de Murcia. http://hdl.handle.net/10201/54519

Rodríguez-Bravo, Blanca; Nicholas, David (2018). “Reputación y comunicación científica: investigadores españoles en el inicio de su carrera". El profesional de la información, v. 28, n. 2, e280203.

https://doi.org//10.3145/epi.2019.mar.03

Russell, Jane M. (2001). “La comunicación científica a comienzos del siglo XXI”. Revista internacional de ciencias sociales, n. 168.

SCI UC3M (2019). Dosieres de seguimiento de impacto en web y medios de comunicación de las noticias de $1+D+i$. Años 2018-2019. e-Archivo de la Universidad Carlos III de Madrid (UC3M). Informes del Servicio de Comunicación Institucional (SCl).

https://e-archivo.uc3m.es/handle/10016/16420

Storer, Norman W. (1966). The social system of science. New York: Holt, Rinehart and Winston. ISBN: 9780030568657

UC3M (2019). La UC3M incrementa sus noticias y vídeos institucionales sobre $1+D+i$. Web de la Universidad, 18 de marzo de 2019.

https://shar.es/a3xvLO 